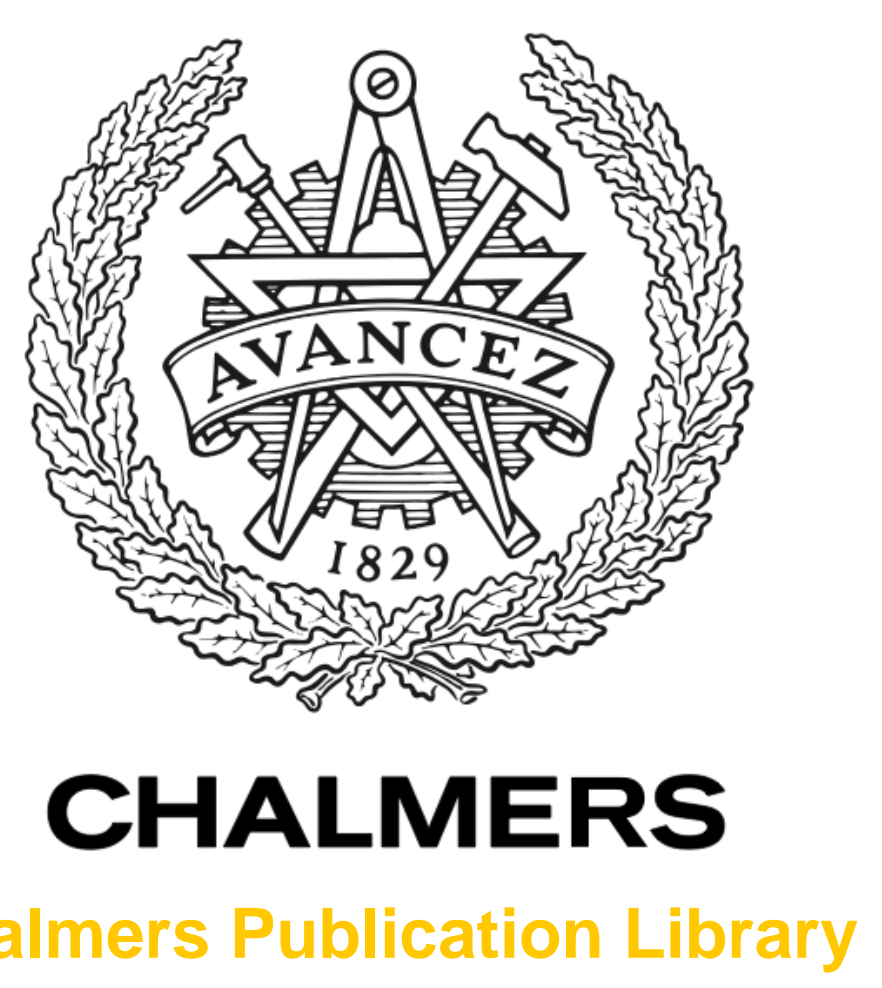

Chalmers Publication Library

Partial Discharges in Motor Wires at PWM Voltages of Different Smoothness

This document has been downloaded from Chalmers Publication Library $(\mathrm{CPL})$. It is the author's version of a work that was accepted for publication in:

Proceedings of 2014 International Symposium on Electrical Insulating Materials (ISEIM 2014), June 1-5, 2014, Niigata City, Japan

Citation for the published paper:

Hammarström, T. ; Bengtsson, T. ; Blennow, J. (2014) "Partial Discharges in Motor Wires at PWM Voltages of Different Smoothness". Proceedings of 2014 International Symposium on Electrical Insulating Materials (ISEIM 2014), June 1-5, 2014, Niigata City, Japan pp. 184187.

http://dx.doi.org/10.1109/ISEIM.2014.6870749

Downloaded from: http://publications.lib.chalmers.se/publication/199843

Notice: Changes introduced as a result of publishing processes such as copy-editing and formatting may not be reflected in this document. For a definitive version of this work, please refer to the published source. Please note that access to the published version might require a subscription. 


\title{
PARTIAL DISCHARGES IN MOTOR WIRES AT PWM VOLTAGES OF DIFFERENT SMOOTHNESS
}

\author{
T. J. Å. Hammarström ${ }^{1 *}$, T. Bengtsson ${ }^{1,2}$, J. Blennow $^{1}$ \\ and S. M. Gubanski ${ }^{1}$ \\ ${ }^{1}$ Department of Materials and Manufacturing Technology \\ Chalmers University of Technology, SE 41296 Gothenburg, Sweden \\ ${ }^{2}$ Also with ABB Corporate Research, SE 72178 Västerås, Sweden \\ *Email:thomas.hammarstrom@chalmers.se
}

\begin{abstract}
Author's recent work has been focused on exploring possibilities for measuring partial discharges (PDs) under the action of pulse width modulated waveforms (PWM), which resulted in a development of an electrical measurement method adopted for such voltages. This solution allows a more flexible PD analysis since voltage shapes appearing in service can be utilized. In this paper, results are presented where several waveforms are applied to quantify the PD properties for motor wire test objects, starting from a non-filtered PWM waveform and continuing with gradually smoothened ones towards an $\mathrm{AC}$ voltage shape. The results suggest that non-smoothed PWM voltage introduces considerably more PDs and with larger magnitudes, which most probably influences the lifetime of the insulation system in all tested cases. Additionally, above a certain level of filtering, the use of lower carrier frequencies implies higher PD exposure, which suggests that the filters used should be evaluated together with the carrier frequency to ensure a longer lifetime of the insulation. It was also observed that the changes in duty cycle reduce the PD density at higher frequencies. This suggests that only applying square voltage waveforms with $50 \%$ duty cycle for PD testing may fail to capture the actual stress inflicted.
\end{abstract}

Keywords: Partial discharges, PWM, square like voltages, measurements, Twisted pair, Motor winding

\section{INTRODUCTION}

The need of a more efficient control of the energy conversion equipment has increased the use of power electronics, particularly in electric motors, where a common approach is today to utilize the so called pulse width modulated voltage (PWM) waveforms. Resent research suggest however that such stresses affect insulation systems negatively, as they yield an increased amount of partial discharges (PDs) due to a presence of high frequency components in the applied voltage. As the phenomenon causes serious degradation and early failures, especially in enamel wire wounded motors, there is therefore a need for monitoring it and developing precaution means. For limiting the degradation, electrical filters are utilized to smooth the PWM pulses, as the frequency content influences the lifetime of insulation systems. Such techniques are however adding costs to the design.

PD measuring techniques, according to IEC 60270, are primarily utilizing the vast difference in frequency content between the applied voltage and the PD signal. A similar detection of partial discharges at a PWM voltage is however difficult as its frequency content is considerably higher than the power frequency. Thus several approaches have been suggested to meet this challenge $[1,2]$. Different voltage patterns have been proposed $[3,4]$ with the aim of recreating similar PD characteristics as within an insulation system exposed to actual PWM voltage. An example is a sinusoidal voltage with superimposed high frequency pulses [5], which altogether are supposed to resemble the stress appearing on the windings in inverter drives due to contributions from reflections of steep voltage fronts. Other waveforms applied are square shaped voltages with different rise times and frequencies but extensively focusing on $50 \%$ duty cycles [6]. To overcome the difficulties arising when using the conventional measuring circuits, a time domain technique has recently been introduced, where the stochastic nature of PDs is employed to separate PD occurrences from the applied voltage [7, 8]. The method allows for exploration of the properties of PDs at varying voltage rise times and in various sources, as demonstrated in [9] by applying semi-square voltage pulses. It is further possible to measure PDs at PWM voltage shapes if the waveform is perfectly stable; however this is most often not the case.

Previous work of the authors has resulted in formulating a hypothesis that, in order to obtain similar PD properties as in the $\mathrm{AC}$ case, the contributions from the carrier frequency remaining in the synthesized waveform should have a lower magnitude than the repetitive extinction voltage (RPEV). [10]. For confirming validity of this hypothesis, the influence of smoothing level was additionally studied at several carrier frequencies. The investigations were performed by keeping peak values constant at all tested voltage levels.

\section{PD DETECTION AT PWM VOLTAGES}

Some characteristics of PWM sources make the technique utilized in [9] less suitable. It is possible, for example, to determine position of PDs with respect to specific polarity reversals, but it is not possible to relate these events to the phase position within the modulated waveform. Thus it becomes impossible to create a phase resolved PD (PRPD) pattern, which makes a direct comparison with sinusoidal conditions difficult. Measurement of PDs under PWM voltage waveforms becomes especially challenging when the relation between the carrier and the modulating frequency is not an even multiplier and the duty cycle changes constantly. In addition, the timing jitter of a PWM pattern complicates the situation further. The challenges to detect PDs at PWM voltages have been met by dividing the captured waveform into parts related to the position of the voltage flanks. Further 
TABLE I. DIFFERENT ELECTRIC FILTER COMPONENTS AND THEIR CHARACTERISTICS.

\begin{tabular}{|c|c|c|c|c|c|}
\hline Level & $\begin{array}{c}\mathrm{R}_{4} \\
(\mathrm{k} \Omega)\end{array}$ & $\begin{array}{c}\mathrm{R}_{5} \\
(\mathrm{k} \Omega)\end{array}$ & $\begin{array}{c}\mathrm{C}_{2} \\
(\mathrm{pF})\end{array}$ & Smoothness & $\mathrm{T}_{\mathrm{r}}(\mu \mathrm{s})$ \\
\hline 1 & 0 & - & - & $0.000075=75 \mu$ & 0.5 \\
\hline 2 & 2 & - & - & $298 \mu$ & 2 \\
\hline 3 & 12 & - & - & $0.0018=1.8 \mathrm{~m}$ & 12 \\
\hline 4 & 100 & 100 & - & $9 \mathrm{~m}$ & 60 \\
\hline 5 & 300 & 100 & - & $13 \mathrm{~m}$ & 88 \\
\hline 6 & 1300 & 100 & - & $45 \mathrm{~m}$ & 300 \\
\hline 7 & 1300 & 100 & 100 & $80 \mathrm{~m}$ & 500 \\
\hline 8 & 2300 & 100 & 100 & 0.12 & 800 \\
\hline
\end{tabular}

a non-smoothed PWM voltage is applied as a separate synchronization channel to relate the smoothed signal to the non-filtered one. Based on this synchronization, the positions between each of the flanks within the synthesized period are detected. These positions are then utilized to divide the captured waveform into separate sub-traces, each stored according to class. The flanks constituting the PWM waveform are classified as either the "Fall" or the "Rise" class dependent on the polarity reversal. Within each class, the time is adjusted to make the voltage step appear at the same time in every sub-trace. When each new sub-trace within a class is adjusted to the previous flanks of the same class, the average value of the traces is calculated and used to eliminate voltage remnants, which enables detection of the PDs within the sub-trace. The detected PDs are then related to two time frames: the first in relation to the nearest voltage flank and the second to the phase position within the modulated frequency. For further details, see [10].

\section{TEST SET-UP}

To detect PDs, an electrical measurement method which enables the quantification of PDs at steep wave fronts was applied, as described in detail in [10]. The main components of the test set-up is shown in figure 1, the test object and the $\mathrm{PD}$ detector is connected to point ' $\mathrm{A}$ '. The reference nonsmoothed waveform is obtained from point "B". Further a bipolar voltage source was used with the possibility to vary the rise times as well as the voltage level. Figure 2 and Table 1 illustrate the test system and the different components utilized to filter the applied voltage waveform to different

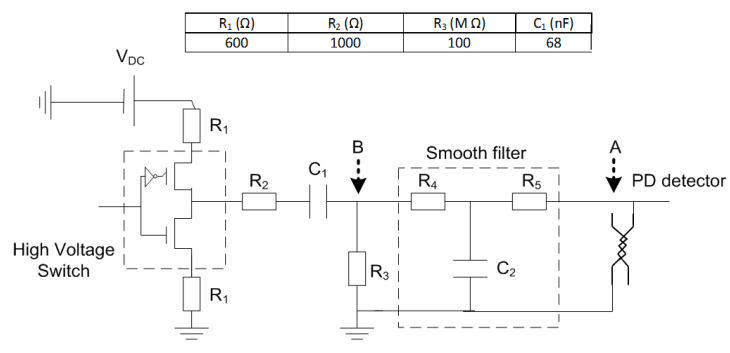

Figure 1 The test set-up including the position of the PD signal detector and the different parameters used to smooth the applied $P W M$ voltage.

The work presented in this publication has been performed within the ELEKTRA programme, project 36137, (with support from the Swedish Energy Agency, Elforsk, ABB AB and Banverket) as well as Chalmers Energy Initiative. smoothness levels. The applied PWM shaped voltage signals had a modulating frequency of $50 \mathrm{~Hz}$ and carrier frequencies of 1,2 , and $3 \mathrm{kHz}$ respectively. Data from 200 cycles were collected at each smoothness level. The smoothness is defined as the ratio between the rise time of the filtered voltage and the sinusoidal waveform [2]:

$$
S=\frac{T_{r_{-} \text {applied }}}{T_{r_{-} \text {sin }}}
$$

\section{MOTOR WINDING INSULATION}

General tests evaluating the performance of random wound motor insulation often employ a twisted wire pair test object [3-5]. This allows the study of shorter rise times as well as gradually smoothed with the test-setup illustrated in figure 1 .

Several investigations of time to breakdown tests have been presented for the traditional winding material using organic polyamide-imide insulation $[1,4]$. A series of investigations presented in [3], suggests factors such as harmonic content of the applied waveform and the wire dimension as influencing the life of polyamide-imide insulated wires considerably. This emphasizes the need to extend the knowledge obtained from time to breakdown analyses, particularly when exposed to rapidly changing waveforms as well as considerably shorter rise times. Preferably, the tests should be performed by applying actual PWM shapes of different frequencies. Other investigations [11] suggest that the life time of corona resistant wire insulation is reduced when going from sinusoidal to square waveform when the same frequency is applied. These tests are however limited to square waves with $50 \%$ duty cycle. Thus, tests applying realistic wave forms, as in actual uses, on adequate types of insulation to further elucidate the PD characteristics have high priority. A natural hypothesis, due to the degrading nature of PDs, is that the PD exposure will be severely influenced by the smoothness of the applied voltage as presented in [2]. Therefore an investigation was performed on twisted pair test objects with a diameter of $1.5 \mathrm{~mm}$ and a $40 \mu \mathrm{m}$ insulation thickness, connected as illustrated in figure 1 with one wire to voltage and the other to ground. This test is supposed to resemble the electric stress appearing in a random wound motor. The test objects were prepared according to the standard requirements [12].

The peak voltage level was chosen to be more than $10 \%$ above the inception voltage level at a $\mathrm{V}_{\mathrm{pp}}$ of $2.5 \mathrm{kV}$, which was kept constant for all smoothness levels and frequencies employed during the first test.

In figure 2 the different PRPD pattern are illustrated for the non-smoothed waveforms for the used carrier frequencies $(1,2$ and $3 \mathrm{kHz})$. It can be observed that the maximum PD magnitude remains at about the same level for all the cases, which implies a higher number of large PDs per trace at increasing carrier frequency. Further is it apparent that a large deviation in duty cycle implies slightly less dense PD pattern, which becomes more noticeable at the higher carrier frequencies. This suggests that a 50\% duty cycle square voltage may have more PDs at about $2 \mathrm{kHz}$ and of higher intensity than a PWM with an equivalent carrier frequency.

For smoothed PWM waveforms, exemplified in figure 3, the PDs will decrease in magnitude and number until the sinusoidal PRPD pattern appears. Additionally, the influence 
of the dips in the synthesized wave form, remnants of voltage steps, is still seen in the PD pattern. This observation is valid although the magnitude of the steps has decreased due to increased smoothness and carrier frequency.

To study how the PD properties are dependent on the smoothness level, some relevant characteristics are presented in figures 4 and 5. Figure 4 illustrates how the maximum PD magnitude depends on the smoothness level. At the lowest smoothness, all the investigated cases give about the same PD magnitude. With increasing smoothness, this maximum magnitude is preserved for the highest carrier frequency until smoothness level 4 is reached, whereas the magnitude decreases immediately for lower carrier frequencies. The highest carrier frequency will additionally cause the largest number of PDs until the same smoothness level 4, is reached as presented in figure 5. A higher carrier frequency then implies an increased PD exposure for lower smoothness levels similarly as observed in [10]. It can be observed that more than one PD per polarity shift appears in each case and thus higher carrier frequency implies increased PD exposure. This is, however, only the case for lower smoothness as seen from figure 5 .

For increasing smoothness, the use of $1 \mathrm{kHz}$ carrier frequency introduces a higher amount of PDs. As is apparent from figure 3 the remaining voltage steps, superimposed on the sinusoidal waveform, are higher for the lowest frequency and thus the probable reason is that large enough voltage steps for PDs to occur remains longer at lower carrier frequency. Thus the choices of filtering as well as carrier
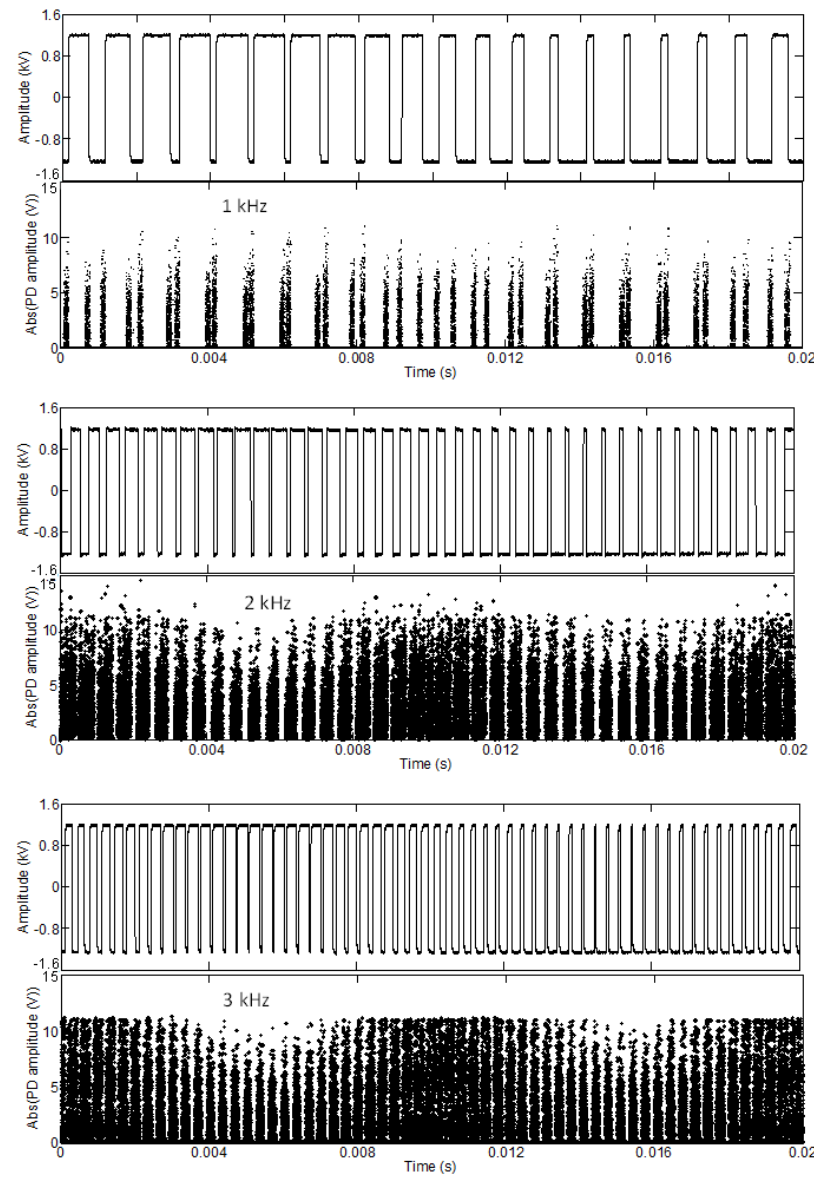

Figure 2 PD distributions for the lowest smoothness. Note that the PD pattern increases in intensity with the carrier frequency. Further note the influence of duty cycle at higher frequency.
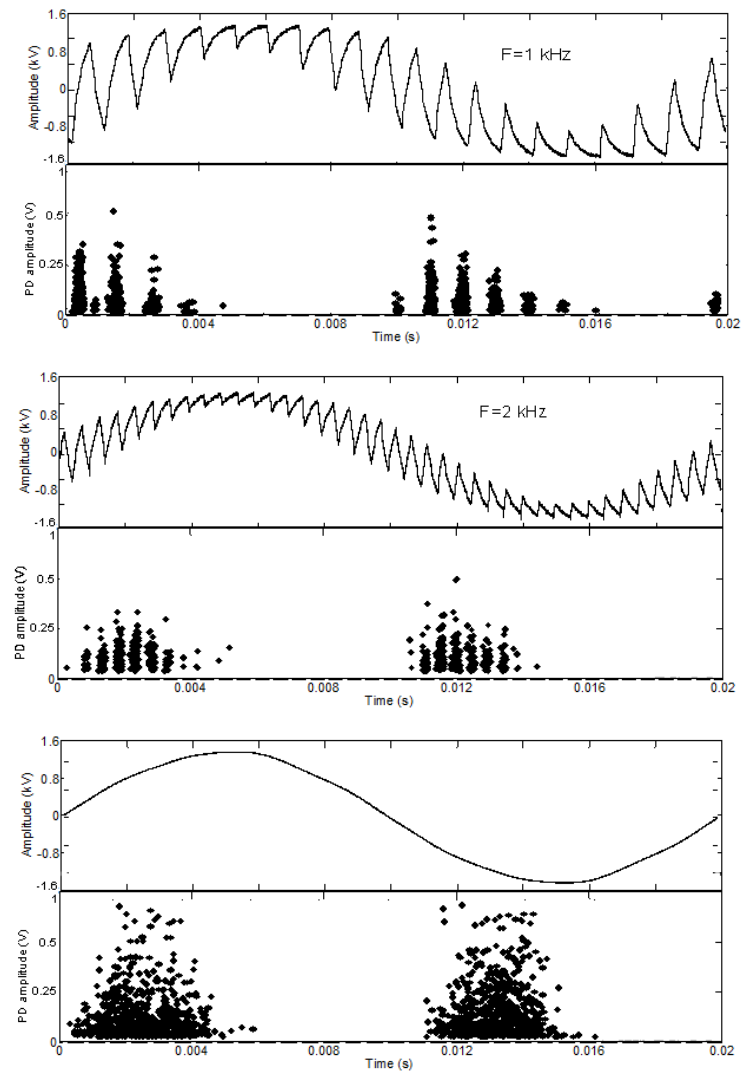

Figure 3 Influence of carrier frequency on PRPDA pattern for constant peak amplitude $V_{p p}$. The two top panels show smoothness 0.12 and the lowest panel sinusoidal voltage, i.e., smoothenss 1 .

frequency are important parameters to consider when designing a suitable insulation system for motor applications. To reach the same level of PD exposure as for sinusoidal waveforms, a smoothness on the vicinity of level 6 is required. A study on how the degradation due to this smoothness differs from the lowest smoothness is thus relevant and presented in the next section.

\section{DEGRADATION TEST}

As a complement to the electrical measurement, two test samples were exposed to identical number of cycles of PWM waveforms with different smoothness. One sample was exposed to the lowest smoothness level in table 1 (level 1) and the other one to a wave shape with the smoothness where the same PD exposure as for sinusoidal conditions were obtained (level 6). The carrier frequency was kept at $1 \mathrm{kHz}$

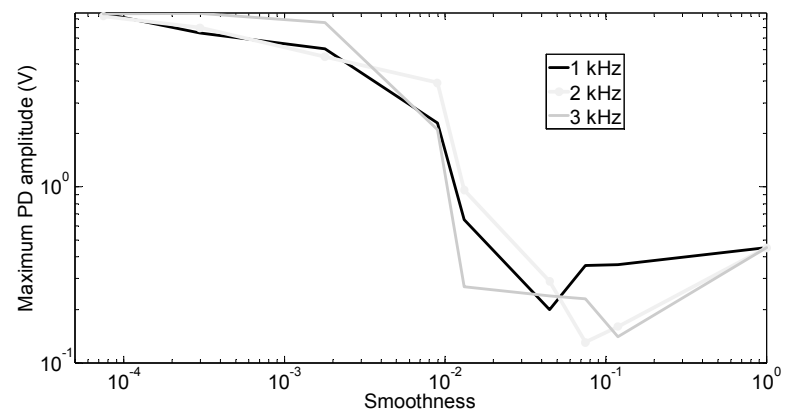

Figure 4 The maximum PD amplitude captured during each cycle at constant $V_{p p}$ value. Note that sinusoidal conditions are reached at smoothness level 5. 


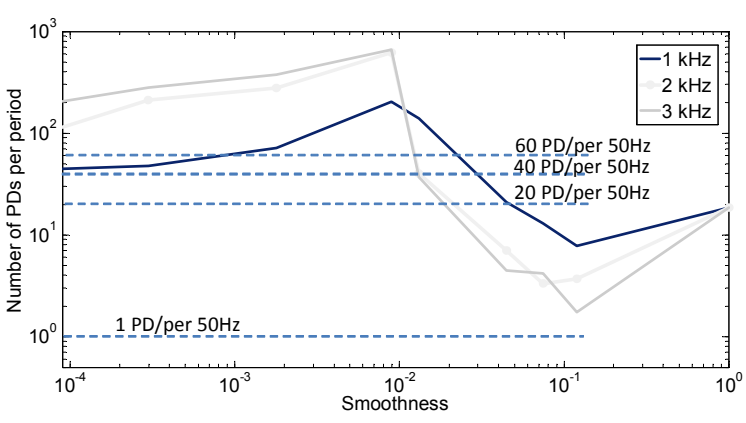

Figure 5 Number of PDs detected for different carrier frequencies and smoothness. Note that for low smoothness the detected number of PDs exceeds the number of polarity shifts within a given period but that this relation disappears for higher smoothness.

for both cases and 8 Mcycles were run for each of the objects. The expected outcome from this test is that, for the lower rise time, the larger number and amplitude of PDs will result in increased damage around the area where the two wires are in contact. The microscope pictures of the surface between the two crossed wires, shown in figures 6 and 7, indeed support this assumption. It is clear that a lower smoothness will indeed erode a larger area which likely will result in a decreased life time. This supports the correlation between observed PD characteristics and level of degradation.

\section{CONCLUSIONS}

The results presented here together with similar

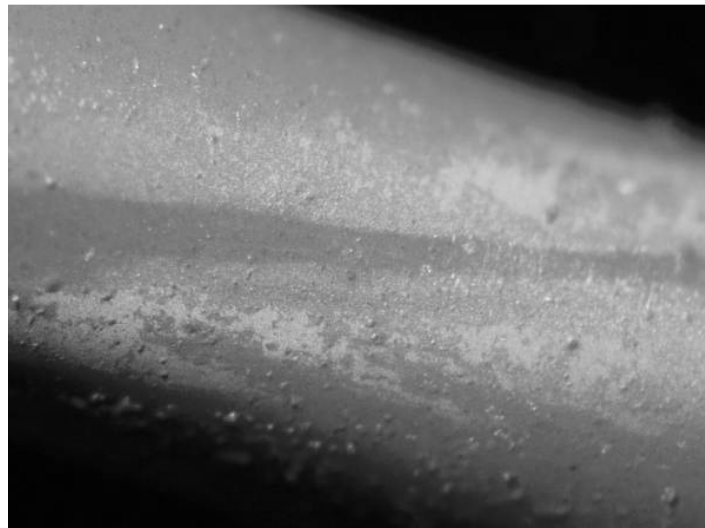

Figure 6 Surface degradation for a wire exposed to 8 Mcycles of PWM waveform with smoothness level 1 and amplitude $2.5 \mathrm{kV}$. Note that the surface outside the contact area is severely more damaged than for the higher smoothness, shown in figure 7.

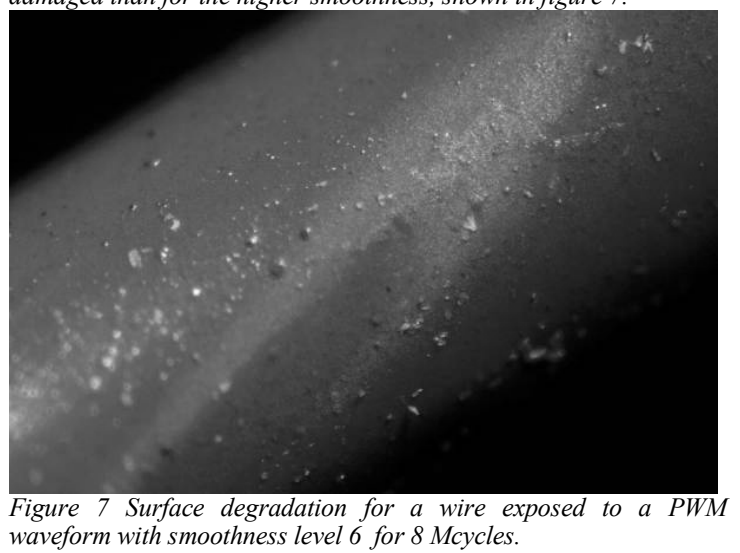

waveform with smoothness level 6 for 8 Mcycles. observations in $[2,9]$ suggest that the base for the suggested relation between rise time and PD exposure seem to be valid in several insulation systems. To conclude these investigations, it is shown that the smoothness indeed affect both the peak amplitude and the number of PDs occurring. Since the PD amplitude is often important for the lifetime of the insulation system, it must be noted that even corona resistant insulation, which is very efficient when applying sinusoidal waveforms, might be less successful at PWM voltages. Here the larger PD magnitudes result in a higher stress level on the outer insulation material. The smoothness limit found in this work can hopefully act as an indication for determining at what wave shape a similar lifetime as under sinusoidal waveforms can be expected. As the low smoothness waveform inflicts higher magnitudes of PDs and an increased surface wear, it is likely that the observed changes in lifetime reported in [2, 3, and 11] are due to increased PD exposure. A limiting smoothness level has been defined above where the PD wear is reduced considerably.

\section{REFERENCES}

[1] G. F. Guastavino, G. Coletti, B. Cerutti and E. Torello, "An Experimental Study About the Weight of the (dv/dt) Factor Characterizing Inverter-like Voltages During Pre-selection Tests on Copper Enameled Wires for Adjustable Speed Drives Motors", 2000 Power Engineering Society Summer Meeting, PP. 2446-2488, Vol. 4, Seattle, WA, USA, 2000.

[2] F. Guastavino, G. Coletti, B. Cerutti and E. Torello, "Partial Discharge Activity in Presence of Pulsed Waveform", IEEE Power Engineering, Society General Meeting, 2008.

[3] B. Florkowska, M. Florkowski, J. Furgal, J. Roehrich and P. Zydron, "The voltage stresses of insulation systems under PWM inverter supplies", IEEE Annual Report Conference on Electrical Insulation and Dielectric Phenomena, pp. 372-375.

[4] D. Fabiani, G. C. Montanari, A. Cavallini and G. Mazzanti, "Relation Between Space Charge Accumulation and Partial Discharge Activity in Enameled Wires under PWM-like Voltage Waveforms", IEEE Transactions on Dielectrics and Electrical Insulation, Vol. 11, No. 3; June 2004.

[5] S. Grzybowski, P. Shrestha and L. Cao, "Electrical Aging Phenomena of XLPE and EPR Cable Insulation Energized by Switching Impulses". International Conference on High Voltage Engineering and Application, Chongquin, China, Nov. 9 -13, 2008.

[6] T. Koltunowicz, A. Cavallini, D. Djairam, G. C. Montanari and J. Smit, "The Influence of Square Voltage Waveforms on Transformer Insulation Break Down Voltage", Conference on Electrical Insulation and Dielectric Phenomena, pp. 48 - 51, Cancun, Mexico, 2011.

[7] E. Lindell, T. Bengtsson, J. Blennow and S.M Gubanski, "Influence of Rise Time on PD Extinction Voltage at Semi-Square Voltage Waveforms", IEEE Transactions on Dielectrics and Electrical Insulation, Vol. 17, Issue 1, pp. 141-148, 2010

[8] T. Hammarström, T. Bengtsson, J. Blennow and S. M Gubanski, "Resonant PD Signal Decoupling Circuit for Rapidly Changing Voltages", Proceedings of the 22st Nordic Insulation Symposium, June 13-15, 2011, Tampere, Finland.

[9] T.Hammarström, T. Bengtsson, J. Blennow and S. M Gubanski, "Evidence Changines in PD Properties with Decreasing Voltage Rise Time in Dielectrically Insulated Cavities". Proceedings of the 17th International Symposium on High Voltage Engineering, ISBN/ISSN 978-3-8007-3364-4, 2011.

[10] T. J Hammarström, T. Bengtsson, J Blennow, S. M Gubanski, "PD Properties at PWM Voltages of Varying Smoothness", IEEE Transactions on Dielectrics and Electrical Insulation, Vol. 20, Issue 6, pp. 2035-2042, December 2013.

[11] B. Florkowska, M. Florkowski, J. Furgal, J. Roehrich, P. Zydron, "Impact of Fast Transient Phenomena on Electrical Insulation Systems", Wydawnitcha AGH, Krakow 2012, ISBN 978-83-74644808

[12] IEC 60851-5 Winding wires - Test methods - Part 5: Elelctrical properties. 